\title{
Brolucizumab: is extended VEGF suppression on the horizon?
}

\author{
Ashish Sharma $^{1}$ - Nilesh Kumar (i) ${ }^{1} \cdot$ Baruch D Kuppermann $^{2} \cdot$ Anat Loewenstein $^{3} \cdot$ Francesco Bandello $^{4}$
}

Received: 5 June 2019 / Accepted: 18 June 2019 / Published online: 16 September 2019

(c) The Royal College of Ophthalmologists 2019

Anti-VEGF therapy has revolutionized the management of neovascular age-related macular degeneration (nAMD) in the last few years $[1,2]$. It has become the first line treatment option for nAMD [3]. However, monthly dosing regimen was being bogged by multiple hospital visits, financial burden to the patient and healthcare system [4]. The researchers have explored newer treatment strategies such as Pro-re-nata (PRN), Treat and Extend (TREX) and three monthly interval (PIER) to extend dosing intervals [4-6]. However, the need of a longer acting anti-VEGF agent was felt.

Ranibizumab port delivery system, Abicipar, Faricimab and Brolucizumab are recent efforts to provide long term VEGF suppression. Amongst these, brolucizumab \{Humanized single chain antibody fragment $(\mathrm{scFv})\}$ has reached closer to marketing approval [7-9]. Recently concluded phase 3 HAWK and HARRIER trials have proven its safety and efficacy in nAMD and the marketing approval has been sought from the FDA by Novartis [10, 11].

Brolucizumab was ideated by ESBATech (ESBATech AG-Schlieren ZH, Switzerland) as ESBA1008 as the smallest unit of a novel anti-VEGF monoclonal antibody i.e. an scFv molecule that binds to all isoforms of VEGF-A and blocks their action. ESBA1008 was developed by grafting complementarity-determining regions of the novel anti-VEGF antibody to a human scFv scaffold [12]. The pre-clinical data revealed that the retina had a 2.2 times

Ashish Sharma

drashish79@hotmail.com

1 Department of Vitreoretina, Lotus Eye Hospital and Institute, Coimbatore, TN, India

2 Gavin Herbert Eye Institute, University of California, Irvine, CA, USA

3 Division of Ophthalmology, Tel Aviv Sourasky Medical Center and Sackler Faculty of Medicine, Tel Aviv University, Israel

4 Department of Ophthalmology, University Vita-Salute, Scientific Institute San Raffaele, Milano, Italy higher exposure to the molecule when compared with ranibizumab. RPE/Choroid complex also had 1.7 times higher exposure. Higher tissue penetrance was attributed to the smaller molecular size ( $28 \mathrm{kDa}$, compared with $48 \mathrm{kDa}$ of ranibizumab, $115 \mathrm{kDa}$ of aflibercept) [13]. The higher penetrance was postulated to give better fluid control across layers of retina resulting in better visual outcomes, which was later proven in HAWK and HARRIER trials [10]. The smaller molecular weight has allowed a higher molar concentration of the drug to be fit into the $50 \mu \mathrm{l}$ with formulations up to $120 \mathrm{mg} / \mathrm{ml} .50 \mu \mathrm{l}$ of $120 \mathrm{mg} / \mathrm{ml}$ formulation has 22 times molar excess than $0.5 \mathrm{mg} / 0.5 \mathrm{ml}$ ranibizumab and 12 times more than $2 \mathrm{mg} / 0.5 \mathrm{ml}$ of aflibercept [14]. The in-vitro studies have shown brolucizumab having affinity to VEGF similar to ranibizumab and higher than bevacizumab [15]. In-vivo studies in primates have revealed that the systemic exit of the drug is significantly less than ranibizumab or aflibercept, possibly due to better tissue uptake of the molecule. The reduced systemic exit can lead to less generation of anti-drug antibody, which play a major role in immunogenicity and treatment failure [16].

The phase $1 / 2$ study evaluated the safety and efficacy of ESBA1008 with a new code- RTH258 and demonstrated non-inferiority of $4.5 \mathrm{mg}$ and $6 \mathrm{mg}$ dosing of RTH258 in central subfoveal thickness improvement compared to $0.5 \mathrm{mg}$ ranibizumab, with the dosing interval safely being extended 30 days beyond the regular monthly interval making it a q8w dosing formulation [13].

Alcon Research (Ft. Worth, TX; Basel, Switzerland) took over further development of RTH258 in phase 2. The phase 2 OSPREY trial compared the molecule with aflibercept in a 56-week study duration on 89 eyes showed non-inferiority compared to aflibercept in a $\mathrm{q} 8 \mathrm{w}$ dosing schedule and supported further development [15, 17].

Alcon Research initiated the phase 3 trials with 2 similarly designed HAWK and HARRIER trials in treatment naïve nAMD patients at 408 sites globally. $3 \mathrm{mg}$ and $6 \mathrm{mg}$ dosing of brolucizumab were compared with $2 \mathrm{mg}$ of aflibercept in 1775 target eyes, whereas HARRIER trial was designed for head to head comparison of 
$6 \mathrm{mg}$ dosing with $2 \mathrm{mg}$ of aflibercept targeting 1049 eyes. The studies were completed in March 2018 with 1082 patients in HAWK and 743 patients in HARRIER trials [10]. The brolucizumab dosing included 3 monthly loading doses at 0,4 and 8 weeks and then every 12 weeks (q12w) injection interval, with an option to switch to $\mathrm{q} 8 \mathrm{w}$ dosing based on disease activity. Aflibercept followed a q8w fixed dose interval schedule. At 48 weeks, the primary outcome, that was non-inferiority of BCVA in patients receiving brolucizumab compared to aflibercept was demonstrated successfully in both $3 \mathrm{mg}$ and $6 \mathrm{mg}$ dosing. $55.6 \%$ of the patients in HAWK and $51 \%$ of the patients in HARRIER trials in $6 \mathrm{mg}$ group and $49.4 \%$ of patients of the $3 \mathrm{mg}$ group maintained q12 $\mathrm{W}$ dosing at the end of 48 weeks. When adjusted for the absence of disease activity during the first $\mathrm{q} 12 \mathrm{w}$ interval, the probability of maintaining the dosing increased up to $85.4 \%$. Brolucizumab resulted in significantly less disease activity and greater central subfoveal thickness reduction at the end of the 16-week period. The 16-week period was an important temporal landmark as till this point, all the arms had similar loading dose schedule and follow-up providing a head to head comparison to aflibercept. The molecule also showed to be significantly better in drying the macular oedema with more reduction in intraretinal fluid/subretinal fluid and sub-RPE fluid at weeks 16, 36 and 48. Serious adverse events were comparable in both groups during the 48-week period and has thus been established for the safety of the drug [10]. The 96-weeks reports are yet to be published after statistical analysis and are anticipated to give a better insight on the dosing requirement, maintaining the visual gain and stability of macular oedema reduction.

Spirited by the promising phase 3 results, Novartis has filed a biological licence approval application with USFDA and has secured an expedited approval for the drug. Brolucizumab is anticipated to be launched for clinical use by the end of 2019 following its USFDA approval [11].

The molecule has in conclusion, shown better promise than existing anti-VEGF options such as better molar dosing, better tissue penetration, less systemic exit, longer antiVEGF suppression, non-inferior BCVA improvement and better anatomical outcome when compared with aflibercept and ranibizumab. The real-world translations of these promises are bound to be scrutinised after the marketing approval, as they have the potential to reduce the healthcare burden by shifting the treatment protocol to $\mathrm{q} 8 \mathrm{w}$ or $\mathrm{q} 12 \mathrm{w}$ dosing interval [7-9, 18].

\section{Compliance with ethical standards}

Conflict of interest The authors declare that they have no conflict of interest.
Publisher's note Springer Nature remains neutral with regard to jurisdictional claims in published maps and institutional affiliations.

\section{References}

1. Brown DM, Michels M, Kaiser PK, Heier JS, Sy JP, Ianchulev T. Ranibizumab versus verteporfin photodynamic therapy for neovascular age-related macular degeneration: two-year results of the ANCHOR study. Ophthalmology 2009; 116:57-65.e5.

2. Mitchell P, Bressler N, Doan QV, Dolan C, Ferreira A, Osborne A, et al. Estimated cases of blindness and visual impairment from neovascular age-related macular degeneration avoided in Australia by ranibizumab treatment. PLoS One. 2014;9. https://www.ncbi. nlm.nih.gov/pmc/articles/PMC4076243/

3. Chong V. Ranibizumab for the treatment of wet AMD: a summary of real-world studies. Eye. 2016;30:270-86.

4. Boyle J, Vukicevic M, Koklanis K, Itsiopoulos C, Rees G. Experiences of patients undergoing repeated intravitreal anti-vascular endothelial growth factor injections for neovascular age-related macular degeneration. Psychol Health Med 2018;23:127-40.

5. Chin-Yee D, Eck T, Fowler S, Hardi A, Apte RS. A systematic review of as needed versus treat and extend ranibizumab or bevacizumab treatment regimens for neovascular age-related macular degeneration. Br J Ophthalmol 2016;100:914-7.

6. Regillo CD, Brown DM, Abraham $\mathrm{P}$, Yue H, Ianchulev $\mathrm{T}$, Schneider S, et al. Randomized, double-masked, sham-controlled trial of ranibizumab for neovascular age-related macular degeneration: PIER study year 1. Am J Ophthalmol. 2008;145:239-248. e5.

7. Campochiaro PA, Marcus DM, Awh CC, Regillo C, Adamis AP, Bantseev V, et al. The port delivery system with ranibizumab for neovascular age-related macular degeneration: results from the randomized phase 2 ladder clinical trial. Ophthalmology. 2019; 126:1141-54.

8. Callanan D, Kunimoto D, Maturi RK, Patel SS, Staurenghi G, Wolf S, et al. Double-masked, randomized, phase 2 evaluation of Abicipar Pegol (an Anti-VEGF DARPin Therapeutic) in neovascular age-related macular degeneration. J Ocul Pharmacol Therapeutics. 2018;34:700-9.

9. Mojumder NI, Khanani AM. Retinal physician-the mechanism of the bi-specific antibody Faricimab. Retinal Phys. 2019. https://www.retinalphysician.com/issues/2019/march-2019/themechanism-of-the-bispecific-antibody-faricimab

10. Dugel PU, Koh A, Ogura Y, Jaffe G, Schmidt-Erfurth U, Brown D, et al. HAWK and HARRIER: phase 3, multicenter, randomized, double-masked trials of brolucizumab for neovascular agerelated macular degeneration. Ophthalmology. 2019; pii: S01616420(18)33018-5.

11. Novartis announces FDA filing acceptance and Priority Review of brolucizumab (RTH258) for patients with wet AMD. Novartis. 2019. https://www.novartis.com/news/media-releases/novartis-a nnounces-fda-filing-acceptance-and-priority-review-brolucizuma b-rth258-patients-wet-amd

12. Gaudreault J, Gunde T, Flyod HS, Ellis J, Tietz J, Binggeli D, et al. Preclinical pharmacology and safety of ESBA1008, a singlechain antibody fragment, investigated as potential treatment for age related macular degeneration. Invest Ophthalmol Vis Sci. 2012;53:3025.

13. Holz FG, Dugel PU, Weissgerber G, Hamilton R, Silva R, Bandello $F$, et al. Single-chain antibody fragment VEGF inhibitor RTH258 for neovascular age-related macular degeneration. Ophthalmology. 2016;123:1080-9. 
14. Tietz J, Spohn G, Schmid G, Konrad J, Jampen S, Maurer P, et al. Affinity and potency of RTH258 (ESBA1008), a novel inhibitor of vascular endothelial growth factor A for the treatment of retinal disorders. Invest Ophthalmol Vis Sci. 2015;56:1501.

15. Singerman LJ, Weichselberger A, Sallstig P. OSPREY trial: randomized, active-controlled, phase II study to evaluate safety and efficacy of RTH258, a humanized single-chain anti-VEGF antibody fragment, in patients with neovascular AMD. Invest Ophthalmol Vis Sci. 2015;56:4801.

16. Sharma A, Kumar N, Kuppermann BD, Francesco B, Loewenstein A. Biotherapeutics and immunogenicity: ophthalmic perspective. Eye. 2019. http://www.nature.com/articles/s41433019-0434-y

17. Dugel PU, Jaffe GJ, Sallstig P, Warburton J, Weichselberger A, Wieland $\mathrm{M}$, et al. Brolucizumab versus aflibercept in participants with neovascular age-related macular degeneration: a randomized trial. Ophthalmology. 2017;124:1296-304.

18. Regeneron Pharmaceuticals Inc. FDA approves EYLEA ${ }^{\circledR}$ (aflibercept) injection sBLA in wet age-related macular degeneration. Regeneron Pharmaceuticals Inc. 2019. https://investor.regeneron. com/news-releases/news-release-details/fda-approves-eylear-a flibercept-injection-sbla-wet-age-related 